\title{
Regulation of tissue responses: the TWEAK/Fn14 pathway and otherTNF/TNFR superfamily members that activate non-canonical NFKB signaling
}

\author{
Linda C. Burkly* \\ Department of Immunology, Biogen Idec, Inc., Cambridge, MA, USA \\ *Correspondence: linda.burkly@biogenidec.com
}

Edited and reviewed by:

Pietro Ghezzi, Brighton and Sussex Medical School, UK

Keywords: non-canonical NFKB signaling, TWEAK, Fn14, TNF superfamily, TNFR superfamily

The immune system mediates tissue responses under both physiological and pathological conditions. In addition to leukocyte subsets, non-hematopoietic tissue cell types actively contribute to shaping tissue responses, including the inflammatory, fibrogenic, and regenerative components. TWEAK and its receptor Fn14, members of the TNF/TNFR superfamily, have emerged as a prominent molecular axis regulating tissue responses (1). Generally leukocyte-derived, TWEAK signals through Fn14, which is highly induced in injured and disease tissues on the surface of parenchymal, vascular, stromal, and progenitor cells, thereby orchestrating a host of tissue-shaping processes, including inflammation, angiogenesis, cell proliferation, and death, and regulation of progenitor cells. Of the downstream signaling pathways, particular attention has been given to the non-canonical NFKB pathway, given that TWEAK induces acute activation of canon-

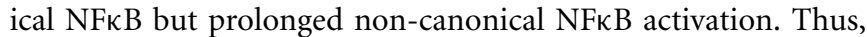
non-canonical NFKB signaling may be a key mechanism underlying TWEAK/Fn14-induced tissue responses. The non-canonical NFKB pathway is known to play a role in immunity and disease pathologies and is typically activated by only a subset of TNFR superfamily members, including Fn14, TNFR2, BAFFR, CD40, LT $\beta R$, and RANK. Thus, there is also broad interest in the role of this subset of TNFR superfamily members and their downstream signaling potentials in the regulation of processes underlying tissue remodeling in health and disease. This Research Topic is addressed in a compilation of 19 expert reviews and 1 original research article.

The TWEAK/Fn14 pathway as an injury-inducible mediator of pleiotropic responses is introduced in a review of the work implicating sustained Fn14 signaling in disease pathogenesis and encompassing the current TWEAK/Fn14-targeting approaches for treatment of human disease (2). Broad relevance to neurological diseases is supported by a basic TWEAK/Fn14 role in regulating the structure and function of the neurovascular unit, thereby regulating blood-brain barrier (BBB) permeability (3). Furthermore, BBB damage appears to be an important component of neuropsychiatric systemic lupus erythematosus, and there is emerging evidence for a role for TWEAK/Fn14 in compromising the BBB in lupus (4). Also relevant to lupus is the pathogenic role of TWEAK/Fn14 in the renal manifestation of lupus nephritis. Indeed, evidence supporting TWEAK/Fn14-mediated pathological mechanisms in contexts of acute kidney injury and chronic kidney diseases is substantial and clinical targeting of TWEAK is ongoing in lupus nephritis (5). Also addressed in this Research Topic is the role of TWEAK/Fn14 in the pathological remodeling underlying other inflammatory diseases, namely cardiovascular diseases and obesity-associated Type- 2 diabetes $(6,7)$, as well as in myocardial remodeling leading to heart failure (8), and a common theme also addressed in these articles is the potential use of soluble TWEAK as a biomarker for cardiovascular diseases. The expression of soluble TWEAK in biological fluids of patients with autoimmune/chronic inflammatory diseases and its potential as a biomarker of these diseases is also more broadly discussed (9). Given that TWEAK has emerged as a major cytokine regulating skeletal muscle biology, two articles are dedicated to its role in muscle wasting and mitochondrial dysfunction, and its complex regulation of myogenesis where distinct roles for canonical versus non-canonical NFKB signaling have been delineated $(10,11)$. Besides Fn14 upregulation in contexts of injury/disease, it is also highly expressed on tumor cells relative to normal tissue making it an attractive therapeutic target. Purcell et al. (12) review the growth inhibitory activity of an Fn14-specific antibody on an array of human tumor cells, differentially dependent on canonical and/or non-canonical NFKB, an approach that is currently being pursued as a novel cancer treatment.

In summary, there is substantial evidence implicating TWEAK/Fn14 in the regulation of physiological and pathophysiological tissue responses, though there is still an incomplete understanding of the role of TWEAK/Fn14-induced canonical versus non-canonical NFKB in these various contexts. Since soluble TWEAK and membrane TWEAK differ in their capacity to induce canonical NFKB, distinct biological responses may reflect spatial and temporal differences in sources of TWEAK (13). New studies continue to inform the understanding of TWEAK-induced signaling, including ubiquitination events that are key to orchestrating canonical and non-canonical NFKB activation (14).

The role of other TNF/TNFR superfamily members in shaping tissue responses in health and disease is also reviewed, concentrating on those that can activate non-canonical NFKB. In a review on TNFa signaling through TNFR2, Faustman and Davis (15) discuss the concept of leveraging TNFR2 agonism to reshape the T cell compartment in autoimmune disease, and to promote tissue regenerative processes. On the other hand, Gardam and Brink (16) review the importance of BAFF/BAFFR-mediated non-canonical 
NFKB signaling in peripheral B cell survival and maturation. Likewise, CD40L-CD40-mediated activation of non-canonical NFкB appears to be critical for $\mathrm{B}$ cell survival and possibly contributes to development of B cell malignancies (17). Both BAFFR and CD40mediated non-canonical NFKB activation in $\mathrm{B}$ cells is restrained by the adaptor protein TRAF3, and the relationship between TRAF3 degradation and non-canonical NF-kB2 activation is delineated in an original research article (18). Beyond $\mathrm{T}$ and $\mathrm{B}$ cell activation, positioning cues are critical for proper immune system development and function. In this regard, LT $\beta$ R plays a critical role in lymph node development and remodeling through its delivery of differentiation signals for reticular networks and vasculature (19). Tissue remodeling in the context of chronic liver diseases also features a prominent role for both LT $\beta$ and TWEAK in the crosstalk between liver progenitor cells and hepatic stellate cells, which can evolve into pathological fibrosis and hepatocellular carcinoma (20). Finally, Walsh and Choi (21) review the RANKLRANK-OPG system, a preeminent player in the bone homeostasis, pathologies including mammary gland tumorigenesis, and in the interplay between bone and the immune system. This collection of expert reviews provides a current perspective of the role of this particular subset of TNF/TNFR family members that can activate non-canonical NFKB signaling in shaping tissue responses in contexts of development, homeostasis, and remodeling.

\section{REFERENCES}

1. Burkly LC. TWEAK/Fn14 axis: the current paradigm of tissue injury-inducible function in the midst of complexities. Semin Immunol (2014) 26:229. doi:10. 1016/j.smim.2014.02.006

2. Cheng E, Armstrong CL, Galisteo R, Winkles JA. TWEAK/Fn14 axis-targeted therapeutics: moving basic science discoveries to the clinic. Front Immunol (2013) 4:473. doi:10.3389/fimmu.2013.00473

3. Yepes M. TWEAK and Fn14 in the neurovascular unit. Front Immunol (2013) 4:367. doi:10.3389/fimmu.2013.00367

4. Stock AD, Wen J, Putterman C. Neuropsychiatric lupus, the blood brain barrier, and the TWEAK/Fn14 pathway. Front Immunol (2013) 4:484. doi:10.3389/ fimmu.2013.00484

5. Poveda J, Tabara LC, Fernandez-Fernandez B, Martin-Cleary C, Sanz AB, Selgas R, et al. TWEAK/Fn14 and non-canonical NF-kappaB signaling in kidney disease. Front Immunol (2013) 4:447. doi:10.3389/fimmu.2013.00447

6. Blanco-Colio LM. TWEAK/Fn14 axis: a promising target for the treatment of cardiovascular diseases. Front Immunol (2014) 5:3. doi:10.3389/fimmu.2014. 00003

7. Vendrell J, Chacon M. TWEAK: a new player in obesity and diabetes. Front Immunol (2013) 4:488. doi:10.3389/fimmu.2013.00488

8. Novoyatleva T, Sajjad A, Engel FB. TWEAK-Fn14 cytokine-receptor axis: a new player of myocardial remodeling and cardiac failure. Front Immunol (2014) 5:50. doi:10.3389/fimmu.2014.00050
9. Bertin D, Stephan D, Khrestchatisky M, Desplat-Jégo S. Is TWEAK a biomarker for autoimmune/chronic inflammatory diseases? Front Immunol (2013) 4:489. doi:10.3389/fimmu.2013.00489

10. Sato S, Ogura Y, Kumar A. TWEAK/Fn14 signaling axis mediates skeletal muscle atrophy and metabolic dysfunction. Front Immunol (2014) 5:18. doi:10.3389/fimmu.2014.00018

11. Enwere EK, Lacasse EC, Adam NJ, Korneluk RG. Role of the TWEAK-Fn14cIAP1-NF-kB signaling axis in the regulation of myogenesis and muscle homeostasis. Front Immunol (2014) 5:34. doi:10.3389/fimmu.2014.00034

12. Purcell JW, Kim HK, Tanlimco SG, Doan M, Fox M, Lambert P, et al. Nuclear factor $\kappa \mathrm{B}$ is required for tumor growth inhibition mediated by enavatuzumab (PDL192), a humanized monoclonal antibody to TweakR. Front Immunol (2014) 4:505. doi:10.3389/fimmu.2013.00505

13. Arana JAC, Seher A, Neumann M, Lang I, Siegmund D, Wajant H. TNF receptor associated factor 1 (TRAF1) is a major target of soluble TWEAK. Front Immunol (2014) 5:63. doi:10.3389/fimmu.2014.00063

14. Vucic D. The role of ubiquitination in TWEAK stimulated signaling. Front Immunol (2013) 4:472. doi:10.3389/fimmu.2013.00472

15. Faustman DL, Davis M. TNF receptor 2 and disease: autoimmunity and regenerative medicine. Front Immunol (2013) 4:478. doi:10.3389/fimmu.2013.00478

16. Gardam S, Brink R. Non-canonical NF- $\kappa B$ signalling initiated by BAFF influences B cell biology at multiple junctures. Front Immunol (2014) 4:509. doi:10.3389/fimmu.2013.00509

17. Hostager BS, Bishop GA. CD40-mediated activation of the NF-kB2 pathway. Front Immunol (2013) 4:376. doi:10.3389/fimmu.2013.00376

18. Lin WW, Hildebrand JM, Bishop GA. A complex relationship between TRAF3 and non-canonical NF- $\kappa$ B2 activation in B lymphocytes. Front Immunol (2013) 4:477. doi:10.3389/fimmu.2013.00477

19. Lu TT, Browning JL. Role of the lymphotoxin/LIGHT system in the development and maintenance of reticular networks and vasculature in lymphoid tissues. Front Immunol (2014) 5:47. doi:10.3389/fimmu.2014.00047

20. Dwyer BJ, Olynyk JK, Ramm GA, Tirnitz-Parker JEE. TWEAK and LT $\beta$ signaling during chronic liver disease. Front Immunol (2014) 5:39. doi:10.3389/fimmu. 2014.00039

21. Walsh MC, Choi Y. Biology of the RANKL-RANK-OPG system. Front Immunol (2014) 5:511. doi:10.3389/fimmu.2014.00511

Conflict of Interest Statement: Linda C. Burkly is a Biogen Idec employee and stockholder.

Received: 09 February 2015; accepted: 17 February 2015; published online: 02 March 2015.

Citation: Burkly LC (2015) Regulation of tissue responses: the TWEAK/Fn14 pathway and other TNF/TNFR superfamily members that activate non-canonical NFK B signaling. Front. Immunol. 6:92. doi: 10.3389/fimmu.2015.00092

This article was submitted to Inflammation, a section of the journal Frontiers in Immunology.

Copyright (c) 2015 Burkly. This is an open-access article distributed under the terms of the Creative Commons Attribution License (CC BY). The use, distribution or reproduction in other forums is permitted, provided the original author(s) or licensor are credited and that the original publication in this journal is cited, in accordance with accepted academic practice. No use, distribution or reproduction is permitted which does not comply with these terms. 